\title{
Non-24-hour sleep-wake syndrome improved by low-dose valproic acid: a case report
}

\author{
This article was published in the following Dove Press journal: \\ Neuropsychiatric Disease and Treatment \\ 13 December 2016 \\ Number of times this article has been viewed
}

\author{
Masatake Kurita ${ }^{1-3}$ \\ Takahiro Moriya ${ }^{2}$ \\ Satoshi Nishino ${ }^{2,4}$ \\ Eishin Hirata ${ }^{4}$ \\ Noriyasu Hirasawa ${ }^{5}$ \\ Yoshiro Okubo ${ }^{3}$ \\ Tadahiro Sato ${ }^{4}$ \\ 'Wakamiya Hospital, Koutokukai, \\ Yoshihara, Yamagata, ${ }^{2}$ Department \\ of Cellular Signaling, Graduate \\ School of Pharmaceutical Sciences, \\ Tohoku University, Sendai, Miyagi, \\ ${ }^{3}$ Department of Psychiatry and \\ Behavioral Science, Graduate \\ School of Medicine, Nippon Medical \\ School, Sendagi, Tokyo, ${ }^{4}$ Sato \\ Hospital, Koutokukai, Kunugizuka, \\ Nanyo, Yamagata, ${ }^{5}$ Laboratory of \\ Pharmacotherapy of Life-Style \\ Related Diseases, Graduate School \\ of Pharmaceutical Sciences, Tohoku \\ University, Sendai, Miyagi, Japan
}

\begin{abstract}
A woman was diagnosed with non-24-hour sleep-wake syndrome and depressive symptoms. Her depressive symptoms did not respond to standard doses of several antidepressants or mood stabilizers. Furthermore, her sleep-wake cycle remained non-entrained despite treatment with a melatonin-related drug, vitamin B12, and phototherapy. Ultimately, her sleep-wake rhythm was restored to a 24-hour pattern with a low dose of valproic acid, and her depressive symptoms tended to improve as a result of synchronization without antidepressants. Low-dose valproic acid appears to be one of the effective means of entraining circadian rhythms in patients with non24-hour sleep-wake syndrome, which in turn likely improves associated depressive symptoms. Keywords: circadian rhythm sleep disorder, mood stabilizers, vitamin B12, melatonin, phototherapy, antidepressants, depression
\end{abstract}

\section{Introduction}

Non-24-hour sleep-wake syndrome is a sleep disorder in which the sleep-wake cycle exceeds 24 hours, and this occurs not only in blind subjects but also in sighted individuals. ${ }^{1,2}$ However, this syndrome hardly occurs in sighted individuals; ${ }^{2}$ the exact mechanism in sighted individuals with non-24-hour sleep-wake syndrome remains to be elucidated. Some evidences suggested that a long circadian period more than the normal range of entrainment is likely a risk factor. ${ }^{3}$ There have been several reports that non-24hour sleep-wake syndrome may be successfully treated with melatonin, ${ }^{4-6}$ vitamin B12, ${ }^{7}$ and phototherapy ${ }^{8}$; however, not all patients respond to these treatments. ${ }^{9}$ Depressive symptoms are more commonly reported among patients with circadian rhythm sleep disorders. The patients with circadian rhythm sleep disorders complained of the interference of the sleep-wake schedule with social obligations. ${ }^{10}$ We report the case of a sighted woman with non-24-hour sleep-wake syndrome and depressive symptoms.

\section{Methods}

Sleep times were recorded at intervals of 30 minutes in a sleep diary by the patient. The circadian period (tau-value) of wake-sleep cycles was determined from the data obtained for a length of 4 consecutive weeks using $\chi^{2}$ periodogram algorithm, and venous blood samples were collected every 4 weeks for assay of the plasma valproic acid concentration. The valproic acid concentration was determined using a VITROS VALP reagent assay kit (Ortho Clinical Diagnostics, Raritan, NJ, USA) according to the manufacturer's instructions. The depressive symptoms were evaluated using the Montgomery-Åsberg Depression Rating Scale (MADRS) ${ }^{11}$; remission of depressive symptoms is defined as a score of $\leq 8$ on the MADRS. ${ }^{12}$ Blood sampling and depression assessment were performed at the same time of day.
Correspondence: Masatake Kurita

Wakamiya Hospital, Koutokukai, 2-15-3

Yoshihara, Yamagata 990-245I, Japan

$\mathrm{Tel}+8 \mathrm{I} 236438222$

Fax $+8 \mid 236441950$

Email kurita@koutoku.or.jp 
A written informed consent was obtained from the patient after the procedures had been fully explained. The study protocol was approved by the Ethics Committee of Sato Hospital, Koutokukai and the Ethics Committee of Graduate School of Pharmaceutical Science, Tohoku University, and standard procedures were followed for clinical trials involving vulnerable participants in Japan. This study was performed according to the ethical standards of the Declaration of Helsinki. The patient also gave written informed consent to publish this case report.

\section{Case presentation}

The patient was a 24-year-old woman who was first referred to our hospital, which is a special outpatient clinic for depression as a major depressive disorder, from another psychiatric hospital. She had already been treated with several antidepressants by the clinicians at the referring psychiatric hospital. Her depressive symptoms, including fatigue and sleep disturbance, interfered with her social obligations; therefore, she could not attend work. Based on the diagnosis provided by the clinicians at the referring psychiatric hospital, we also began treatment for depression. Based on her treatment response, however, we found that she did not have a conventional sleep disorder and instead suspected that she had non24-hour sleep-wake syndrome. The patient had kept a sleep diary over the past 5.5 years, starting from 2008 (Figure 1).
This diary showed that her sleep-wake cycle free-ran on a regular period of 24.55 hours \pm standard deviation 0.30 hours ( $n=47$ periods, where 1 period is 4 weeks), consistent with a diagnosis of non-24-hour sleep-wake syndrome. None of her symptoms had responded to treatment with standard doses of paroxetine (10-30 mg/day), fluvoxamine (25-75 mg/day), imipramine (5-25 mg/day), amitriptyline (20 mg/day), milnacipran (12.5-25 mg/day), mirtazapine (15-30 mg/day), duloxetine (20 mg/day), or amoxapine (2-8 mg/day). Her mean MADRS score over the same period was $17.0 \pm 12.3$ points ( $n=47$, Figure 2$)$, and was unstable during episodes of non-24-hour sleep-wake syndrome.

Although the patient was treated with generally accepted therapeutic doses of mood stabilizers such as lithium (200-600 mg/day), lamotrigine (25-75 mg/day), and valproic acid (400-800 $\mathrm{mg} /$ day), these were ineffective for treating depressive symptoms and non-24-hour sleepwake syndrome. Additionally, her sleep disorder did not respond to 2- or 4-week trials of hypnotics. Ramelteon is an MT1/MT2 melatonin receptor agonist with greater affinity for melatonin receptors and a longer half-life than melatonin. ${ }^{13,14}$ The initial dosage of ramelteon was $8 \mathrm{mg} /$ day for 3 weeks. But I had to reduce ramelteon (2-4 mg/day) as she appealed to me to reduce the dosage for depressive symptoms such as lassitude. Any dosage of ramelteon was ineffective for the patient suffering from non-24-hour

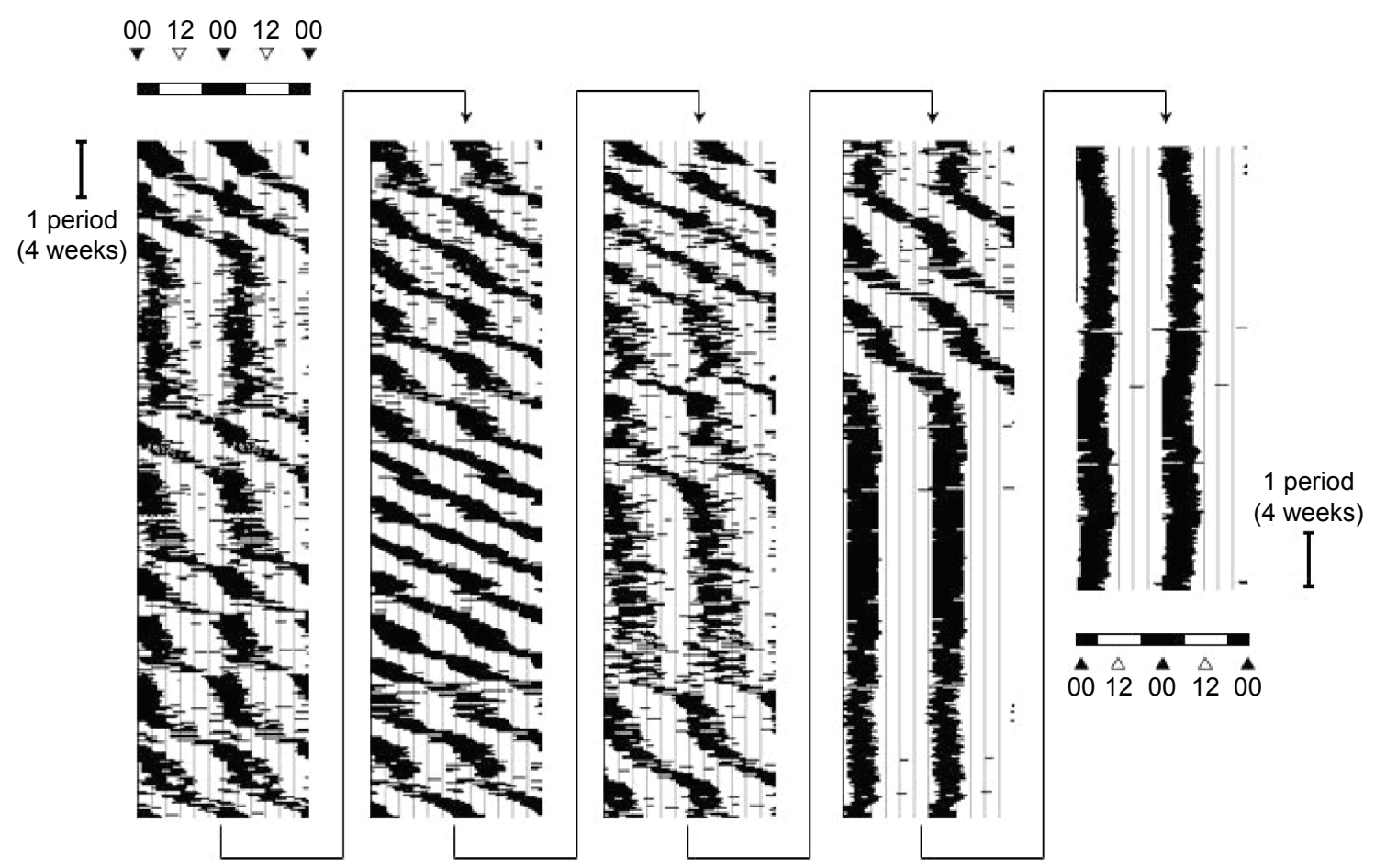

Figure I Double-plotted sleep-awake cycle of a patient.

Note: The times of sleep are shown as black bars and are double-plotted for clarity. 

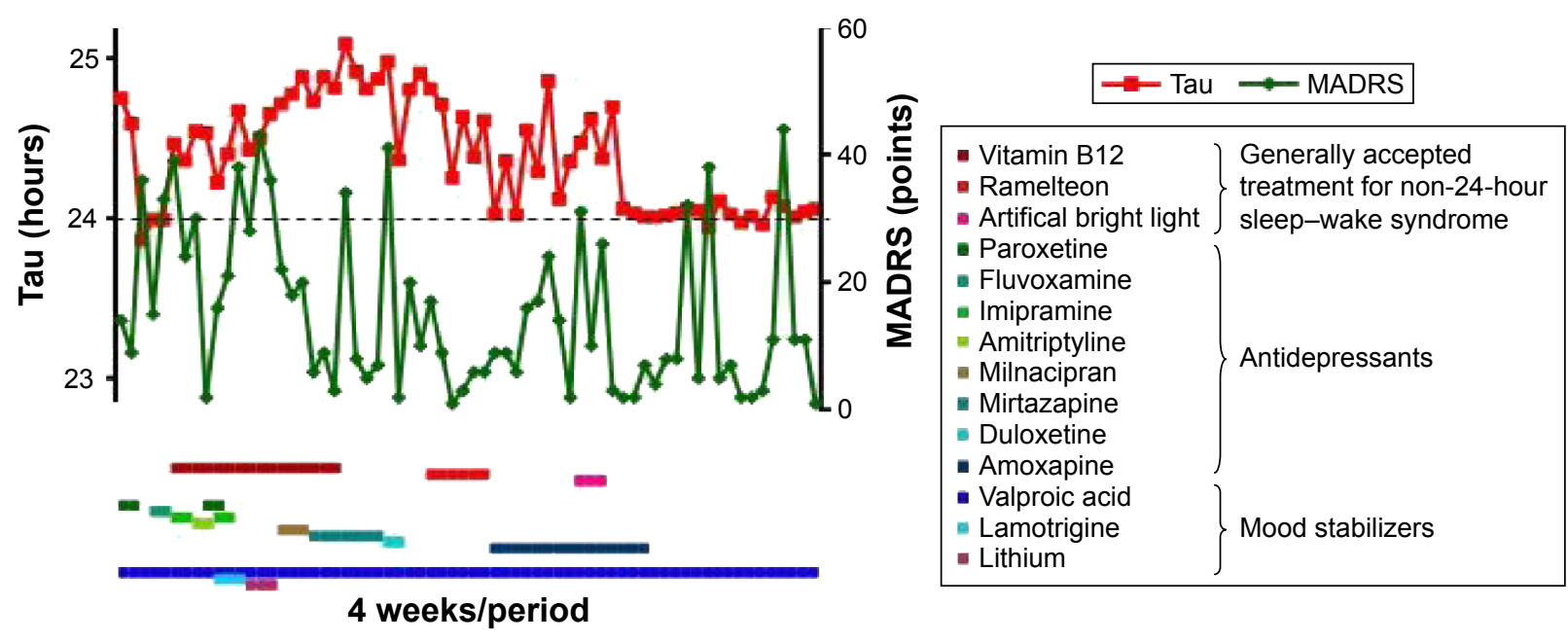

Figure 2 Circadian rhythms (tau-value: red), depressive symptoms (MADRS: green), and drug history (lines).

Notes: Tau-values were determined from actogram and periodogram data every 4 weeks. Remission of depressive symptoms is defined as a score $\leq 8$ on the MontgomeryÅsberg Depression Rating Scale (MADRS).

sleep-wake syndrome. Non-pharmacologic measures, including several treatments of phototherapy (20,000 lux) and efforts to improve sleep hygiene by avoiding daytime sleeping, also failed (Figure 2). The patient continued to complain of difficulty in falling asleep and waking, and reported persistent anxiety, irritation, and fatigue. Laboratory investigations, including serum hormone and immunologic profiles, and magnetic resonance imaging of the brain were normal. Although treatment with valproic acid at the generally accepted dose was ineffective, we considered that treatment with low-dose valproic acid may achieve complete entrainment to a 24-hour sleep cycle. The dose of valproic acid during synchronization was $200 \mathrm{mg}$ /day. The patient's sleep diary during this period showed that the 24-hour sleep-wake cycle synchronized to $24.03 \pm 0.05$ hours $(n=19$ periods, Figure 3$)$ at a valproic acid concentration of

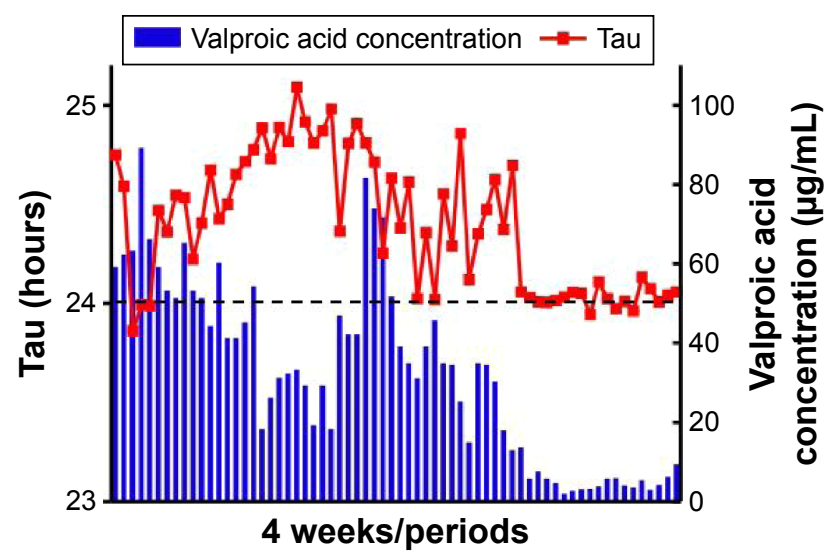

Figure 3 Valproic acid concentration (blue bars) and circadian rhythms throughout (red points) the course of treatment.

Abbreviation: VPA, valproic acid.
$5.0 \pm 2.7 \mu \mathrm{g} / \mathrm{mL}$. The mean MADRS score dropped down to $10.7 \pm 12.7(n=19)$ during synchronization without antidepressants, although the improvement was not statistically significant $\left(F_{2,65}=0.487, P=0.066\right.$, Student's $t$-test $)$. Her sleep onset time, wakeup time, and sleep time were 1:04 $\mathrm{AM} \pm 64$ minutes, 9:26 $\mathrm{AM} \pm 81$ minutes, and 9.3 \pm 1.1 hours during synchronization, respectively. By communicating with the patient, we learned that her symptoms of fatigue and non-24-hour sleep-wake syndrome relapsed because she discontinued the low-dose valproic acid. Thereafter, she restarted treatment with the low-dose valproic acid in an attempt to control her symptoms. However, as she did not visit our hospital during this time, we could not evaluate the changes in the valproic acid concentration.

\section{Discussion}

Valproate is a mood stabilizer with a broad range of actions, but its mechanism of action remains incompletely understood. Valproic acid and divalproex (a combination of sodium valproate and valproic acid) have been licensed for the treatment of bipolar disorder since the 1990s. ${ }^{15}$ The therapeutic range of valproic acid for bipolar disorder is $50-125 \mathrm{mg} / \mathrm{L}$ $(350-850 \mu \mathrm{M}) .{ }^{15}$ Valproate is associated with increased levels of acetylated histones via its action as a histone deacetylase inhibitor. ${ }^{16}$ A particularly interesting finding is that the bimodal effect of valproate resulted in a difference in the transcription of tyrosine hydroxylase at low and high concentrations. ${ }^{17}$ Consequently, the pharmacologic effects of low-dose valproic acid may differ from those of the generally accepted therapeutic dose. Although whether changes in the transcription of tyrosine hydroxylase contributed to the improvement in this patient's non-entrained sleep remains 
unclear, it is notable that the generally accepted therapeutic dose range of valproic acid was ineffective for the treatment of her non-24-hour sleep-wake syndrome.

Our patient had non-24-hour sleep-wake syndrome, which is not a conventional sleep disorder. In such situations, patients may be stressed by daytime sleepiness. This may result in the development of depressive symptoms, such as anxiety, irritation, and fatigue. Non-24-hour sleep-wake syndrome is rare; therefore, without a sleep diary, even expert psychiatrists have difficulty diagnosing this condition. In fact, the patient in this case was referred to our hospital for the treatment of major depressive disorders. Her sleep had not been entrained and her depressive symptoms had not been alleviated by several antidepressants and mood stabilizers, including generally accepted therapeutic doses of valproic acid. Antidepressants or mood stabilizers may not be effective for treating depressive symptoms when a patient is affected by non-24-hour sleep-wake syndrome. In this case, a therapeutic response was observed only when low-dose valproic acid was administered. The relapse and remission of symptoms that occurred when the patient discontinued and restarted valproic acid therapy on her own initiative gives us confidence that this was a genuine therapeutic effect.

The patient's depressive symptoms tended to improve, and the MADRS score during synchronization of her sleep-wake cycle improved in association with the remission of her symptoms. Additionally, the patient experienced several episodes of remission during synchronization without antidepressants; therefore, it may be important to note that her depressive symptoms did not fully improve by synchronization. Another explanation for the incomplete response of her depressive symptoms is that regular social stresses (such as work) and the possibility that she had another sleep disorder - sleepphase syndrome ${ }^{18}$ (as revealed by her sleep diary) - were an influence. This might have accounted for her incomplete remission or lack of significant improvement. Moreover, her depressive symptoms did not respond to doses of several antidepressants during episodes of her non-24-hour sleep-wake syndrome, and the patient had several periods of remission during synchronization without antidepressants. Therefore, the improvement in her depressive symptoms may have been secondary to the improvement in her non-24-hour sleep-wake syndrome. Although melatonin has been shown to improve depression in patients with circadian rhythm sleep disorders, ${ }^{19}$ major depressive disorders should be carefully differentiated from circadian rhythm sleep disorders with depression. In addition, circadian rhythm sleep disorders should be carefully differentiated from conventional sleep disorders by a sleep diary. In this case, treatment of the patient with low dose was quite accidental. It was because of self-adjusting dose by the patient. Any circadian rhythm sleep disorder requires continuous treatment. ${ }^{8}$ Therefore, it should be considered difficult to conclude that the previous bright light treatment had a carryover effect on entraining circadian rhythm.

\section{Conclusion}

The patient with sleep-wake syndrome was successfully treated and her depressive symptoms tended to improve by treatment with low-dose valproic acid. Low-dose valproic acid therapy should be considered for patients with non24-hour sleep-wake syndrome resistant to conservative management strategies and treatment with melatonin-related drugs, vitamin B12, and phototherapy. Further research will be needed to illuminate the therapeutic benefits and mechanism of action of valproic acid in patients with non-24-hour sleep-wake syndrome. Effects of low-dose valproic acid may be a clue used to solve the mechanism in sighted individuals with non-24-hour sleep-wake syndrome. We hope that many researchers will treat the circadian rhythm sleep disorders using low-dose valproic acid in the future.

\section{Acknowledgments}

We thank the study participant, without whom this study could not have been accomplished, as well as the following psychiatrists and staff of Sato Hospital and Wakamiya Hospital, Koutokukai: Yukio Numata, MD; Kayoko Suga; Kazuo Kenmi, MD, PhD; Asao Hasegawa, MD; Masaaki Mitomo, MD; Yukihiro Takeuchi, MD, PhD; Hiroshi Yamamoto, MD; Maiko Kato, MD; and Kenji Takeyoshi, MD, PhD.

This study was supported by funding from Koutokukai and JSPS KAKENHI Grant Number JP26461709. The study did not receive funding from any pharmaceutical corporations.

\section{Disclosure}

The authors report no conflicts of interest in this work.

\section{References}

1. Miles LE, Raynal DM, Wilson MA. Blind man living in normal society has circadian rhythms of 24.9 hours. Science. 1977;198(4315): 421-423.

2. Weber AL, Cary MS, Connor N, Keyes P. Human non-24-hour sleepwake cycles in an everyday environment. Sleep. 1980;2(3):347-354.

3. Uchiyama M, Lockley SW. Non-24-hour sleep-wake rhythm disorder in sighted and blind patients. Sleep Med Clin. 2015;10(4):495-516.

4. Akaboshi S, Inoue Y, Kubota N, Takeshita K. Case of a mentally retarded child with non-24 hour sleep-wake syndrome caused by deficiency of melatonin secretion. Psychiatry Clin Neurosci. 2000;54(3):379-380.

5. Kato M, Kajimura N, Sekimoto M, Watanabe T, Takahashi K. Melatonin treatment for rhythm disorder. Psychiatry Clin Neurosci. 1998;52(2): 262-263. 
6. McArthur AJ, Lewy AJ, Sack RL. Non-24-hour sleep-wake syndrome in a sighted man: circadian rhythm studies and efficacy of melatonin treatment. Sleep. 1996;19(7):544-553.

7. Kamgar-Parsi B, Wehr TA, Gillin JC. Successful treatment of human non-24-hour sleep-wake syndrome. Sleep. 1983;6(3):257-264.

8. Watanabe T, Kajimura N, Kato M, Sekimoto M, Hori T, Takahashi K. Case of a non-24 h sleep-wake syndrome patient improved by phototherapy. Psychiatry Clin Neurosci. 2000;54(3):369-370.

9. Yamadera H, Takahashi K, Okawa M. A multicenter study of sleepwake rhythm disorders: therapeutic effects of vitamin B12, bright light therapy, chronotherapy and hypnotics. Psychiatry Clin Neurosci. 1996;50(4):203-209.

10. Kanathur N, Harrington J, Lee-Chiong T Jr. Circadian rhythm sleep disorders. Clin Chest Med. 2010;31(2):319-325.

11. Montgomery SA, Asberg M. A new depression scale designed to be sensitive to change. Br J Psychiatry. 1979;134:382-389.

12. Mittmann N, Mitter S, Borden EK, Herrmann N, Naranjo CA, Shear NH. Montgomery-Asberg severity gradations. Am J Psychiatry. 1997;154(9): 1320-1321.

13. Zee PC, Wang-Weigand S, Wright KP Jr, Peng X, Roth T. Effects of ramelteon on insomnia symptoms induced by rapid, eastward travel. Sleep Med. 2010;11(6):525-533.
14. Neubauer DN. A review of ramelteon in the treatment of sleep disorders. Neuropsychiatr Dis Treat. 2008;4(1):69-79.

15. Haymond J, Ensom MH. Does valproic acid warrant therapeutic drug monitoring in bipolar affective disorder? Ther Drug Monit. 2010;32(1): $19-29$.

16. Kim HJ, Rowe M, Ren M, Hong JS, Chen PS, Chuang DM. Histone deacetylase inhibitors exhibit anti-inflammatory and neuroprotective effects in a rat permanent ischemic model of stroke: multiple mechanisms of action. J Pharmacol Exp Ther. 2007;321(3):892-901.

17. D’Souza A, Onem E, Patel P, La Gamma EF, Nankova BB. Valproic acid regulates catecholaminergic pathways by concentration-dependent threshold effects on TH mRNA synthesis and degradation. Brain Res. 2009; 1247:1-10.

18. Medicine AAoS. The International Classification of Sleep Disorders, Revised: Diagnostic and Coding Manual. Chicago, IL: American Academy of Sleep Medicine; 2001:128-133.

19. Rahman SA, Kayumov L, Shapiro CM. Antidepressant action of melatonin in the treatment of delayed sleep phase syndrome. Sleep Med. 2010;11(2):131-136.
Neuropsychiatric Disease and Treatment

\section{Publish your work in this journal}

Neuropsychiatric Disease and Treatment is an international, peerreviewed journal of clinical therapeutics and pharmacology focusing on concise rapid reporting of clinical or pre-clinical studies on a range of neuropsychiatric and neurological disorders. This journal is indexed on PubMed Central, the 'PsycINFO' database and CAS,

\section{Dovepress}

and is the official journal of The International Neuropsychiatric Association (INA). The manuscript management system is completely online and includes a very quick and fair peer-review system, which is all easy to use. Visit http://www.dovepress.com/testimonials.php to read real quotes from published authors. 